\title{
Supplementation with a juice powder concentrate and exercise decrease oxidation and inflammation, and improve the microcirculation in obese women: randomised controlled trial data
}

\author{
Manfred Lamprecht ${ }^{1,2 *}$, Georg Obermayer ${ }^{1,2}$, Kurt Steinbauer $^{3}$, Gerhard Cvirn $^{1}$, Lidija Hofmann ${ }^{4}$, \\ Gerhard Ledinski ${ }^{1}$, Joachim F. Greilberger ${ }^{1,5}$ and Seth Hallstroem ${ }^{1}$ \\ ${ }^{1}$ Institute of Physiological Chemistry, Centre for Physiological Medicine, Medical University of Graz, Harrachgasse 21/II, \\ 8010 Graz, Austria \\ ${ }^{2}$ Institute of Nutrient Research and Sport Nutrition, Petersbergenstrasse 95b, 8042 Graz, Austria \\ ${ }^{3}$ SportchirurgiePlus, Centre for Individual Sport Medicine and Surgery, Berthold Linderweg 15, 8047 Graz, Austria \\ ${ }^{4}$ FH JOANNEUM, University of Applied Sciences, Eggenberger Allee 11, 8020 Graz, Austria \\ ${ }^{5}$ Institute of Laboratory Sciences, Dr Greilberger GmbH, Hauptstrasse 140, 8301 Laßnitzhöhe, Austria \\ (Submitted 18 October 2012 - Final revision received 5 March 2013 - Accepted 5 March 2013 - First published online 16 April 2013)
}

\section{Abstract}

Obesity and sedentary lifestyle are associated with increased oxidative stress, inflammation and vessel dysfunction. Previous research has shown that an encapsulated fruit/berry/vegetable juice powder (FBV) supplement or controlled exercise training improve the markers of redox biology, low-grade inflammation and circulation. The aim of the present study was to assess the effects of 8 weeks of supplementation with FBV or placebo, and a single bout of controlled walking on the markers of oxidation, inflammation and skin capillary microcirculation in forty-two obese pre-menopausal women (41 (SD 5) years, non-smokers and BMI 34.5 (sD 3.8 ) $\mathrm{kg} / \mathrm{m}^{2}$ ) using a randomised, double-blind, placebo-controlled design. All assessments were made before and after 8 weeks of capsule supplementation, and preand post-30 min of controlled treadmill walking at $70 \%$ of $\mathrm{VO}_{2 \max }$. Venous blood was collected for the determination of carbonyl proteins (CP), oxidised LDL (ox-LDL), total oxidation status (TOS) of lipids, malondialdehyde, TNF- $\alpha$ and IL- 6 . Capillary blood flow, $\mathrm{O}_{2}$ saturation of $\mathrm{Hb}\left(\mathrm{SO}_{2} \mathrm{Hb}\right)$ and the relative concentration of $\mathrm{Hb}(\mathrm{rHb})$ were assessed at a $2 \mathrm{~mm}$ skin depth. Following 8 weeks of supplementation, compared with placebo, the FBV group had a significant $(P<0.05)$ reduction in CP, Ox-LDL, TOS and TNF- $\alpha$, and a significant increase in blood flow, $\mathrm{SO}_{2} \mathrm{Hb}$ and $\mathrm{rHb}$. Independent of supplementation, moderate exercise significantly increased blood flow and $\mathrm{rHb}$, with a trend towards increased $\mathrm{SO}_{2} \mathrm{Hb}$. Compared with placebo, 8 weeks of supplementation with FBV decreased the markers of systemic oxidation and inflammation. Both FBV supplementation and a single walking bout improved the markers of the microcirculation in these obese women.

Key words: Dietary supplements: Exercise and obesity: Oxidation and inflammation: Skin microcirculation

In recent decades, increasing prevalence of obesity has become a serious public health concern. Suboptimal dietary habits coupled with a sedentary lifestyle are thought to be major contributors to this situation. Overweight and obesity are associated with irregularities in redox homeostasis, imbalanced pro-inflammatory and anti-inflammatory states and microcirculatory dysfunction ${ }^{(1-3)}$. Reactive Oxygen and Nitrogen species and pro-inflammatory cytokines from both visceral and subcutaneous fat compartments are implicated in increased cardiometabolic disease risk $^{(4,5)}$. Recent research has shown that obese people have structural and functional alterations in skin microcirculation, which are proportional to the increase in the degree of global and central obesity ${ }^{(6)}$. Hence, diets rich in antioxidants and anti-inflammatory nutrients, as well as physical exercise, are of interest to combat some of the detrimental side effects of overweight and obesity.

It has been reported that increased consumption of fruits and vegetables improves the body's antioxidant and antiinflammatory capacities ${ }^{(7-9)}$. Nutraceuticals providing phytochemicals and vitamins, such as an encapsulated fruit and

Abbreviations: CP, carbonyl proteins; FBV, fruit, berry and vegetable juice powder concentrate; MDA, malondialdehyde; ox-LDL, oxidised LDL; rHb, relative $\mathrm{Hb}$ concentration; $\mathrm{SO}_{2} \mathrm{Hb}, \mathrm{O}_{2}$ saturation of $\mathrm{Hb}$; TOS, total oxidation status of lipids; $\mathrm{VO}_{2 \text { max }}$, maximum $\mathrm{O}_{2}$ uptake.

*Corresponding author: Dr M. Lamprecht, fax +43 3163809610, email manfred.lamprecht@medunigraz.at 
vegetable juice powder concentrate, have also demonstrated beneficial effects on the markers of oxidative stress, inflammation and skin microcirculation ${ }^{(10-12)}$.

Similar effects are also observed with exercise. Regular exercise training and single bouts of exercise improve redox biology, exert anti-inflammatory effects and are able to enhance the microcirculation in different populations, including overweight subjects ${ }^{(13-16)}$.

However, the influence of both exercise and nutrient supplementation on oxidation, inflammation and skin microcirculation in a target group of obese women has not been established.

Thus, the primary objective of the present study was (1) to explore the effects of a fruit, berry and vegetable juice powder concentrate (FBV) on oxidation, inflammation and skin microcirculation, compared with placebo. The secondary and tertiary goals were (2) to evaluate whether a single bout of defined walking exercise - with or without the FBV treatment - affects the capillary microcirculation from the skin surface to a $2 \mathrm{~mm}$ depth, and (3) to evaluate whether a defined model of walking exercise generates oxidative stress - with or without the FBV treatment - in a cohort of obese but otherwise healthy pre-menopausal women.

\section{Experimental methods}

\section{Study population}

A total of forty-two overweight and obese pre-menopausal women participated in the present trial. Inclusion criteria were as follows: female; age 35-50 years; regular menses; able to participate in walking exercise; non-smokers; sedentary work and lifestyle; BMI between 28 and $40 \mathrm{~kg} / \mathrm{m}^{2}$; no dietary or nutritional supplement use within the 4 weeks before the first exercise test. Exclusion criteria included the following: smokers; women who failed exercise eligibility testing - as described by the Austrian and German standards in sports medicine ${ }^{(17)}$; chronic or excessive alcohol consumption; pregnancy and/or lactation; recent surgery or illness; diabetes; dyslipidaemia; current participation in a weight management programme; diagnosis of osteoporosis or osteopenia; current use of any medication known to significantly influence inflammation, redox biology or haemostasis. In addition to these inclusion and exclusion criteria, a standard blood chemistry panel, exercise echocardiography and maximum $\mathrm{O}_{2}$ uptake $\left(\mathrm{VO}_{2 \mathrm{max}}\right)$ were determined in all women to confirm general health before study enrolment. All subjects also completed a medical history and a physical activity/well-being questionnaire.

\section{Ethical aspects, recruitment and randomisation}

The present study was conducted according to the guidelines laid down in the Declaration of Helsinki, and all procedures involving human subjects were approved by the Ethical Review Committee of the Medical University of Graz, Austria. All subjects provided written informed consent before participating in the present investigation. The trial was registered at www.clinicaltrials.gov (identifier no. NCT01476033).
The study focused on office workers and was announced in local newspapers. A telephone screening conducted by study staff resulted in fifty-nine volunteers for further eligibility testing. Among these volunteers, forty-four women met the inclusion and exclusion criteria and were enrolled.

Subjects were randomised in blocks of six and sequentially numbered (www.randomization.com). To guarantee a balanced BMI distribution between the groups (FBV or placebo), we conducted stratification via BMI rank statistics. The randomisation code was held by a third party (Union of Sport and Exercise Scientists Austria) and provided for statistical analyses of the complete dataset.

\section{Study design and time schedule}

This was a randomised, double-blind, placebo-controlled study. All eligibility testing was finalised 4 weeks before the baseline controlled walking bout. On that morning, a standardised breakfast ( $2-3 \mathrm{~h}$ before exercise) was provided. Then, each subject came to the laboratory to perform her $30 \mathrm{~min}$ exercise test at an intensity of $70 \%$ of individual $\mathrm{VO}_{2 \max }$. After the test, the investigator dispensed the randomised capsule supply according to the subject's BMI ranking. Following 8 weeks of capsule supplementation as directed, they returned their remaining capsules and the same test procedure was repeated. All subjects were checked by a physician before each exercise test. The walking tests were scheduled between days 10 and 20 of the menstrual cycle.

\section{Dietary parameters}

Subjects were instructed to maintain their habitual diet and lifestyle during the 8-week study and to duplicate their diet before each exercise testing/blood collection appointment, as described below. Before the first 30 min walking test, subjects completed a $7 \mathrm{~d}$ food record to assess nutrient intake. Subjects subsequently received copies of their $7 \mathrm{~d}$ diet records and were instructed to replicate the diet before the second exercise test. The standardised breakfast was served $2-3 \mathrm{~h}$ before both exercise tests to limit nutrient variation due to self-selection on the morning scheduled for blood collection. The standardised breakfast consisted of $250 \mathrm{ml}$ low-fat yogurt, $10 \mathrm{~g}$ butter, $20 \mathrm{~g}$ jam or honey, $50 \mathrm{~g}$ rye-wheat bread and $500 \mathrm{ml}$ of plain water, providing $1500 \mathrm{~kJ}, 13 \mathrm{~g}$ protein, $47 \mathrm{~g}$ carbohydrate and $13 \mathrm{~g}$ fat. Diet records were analysed for total energy, protein, carbohydrate, fat, cholesterol, fibre, water, alcohol and several vitamins, minerals and fatty acids using Opti Diet software $5.0(\mathrm{GOEmbH})$.

\section{Study capsules}

Women randomised to the FBV group ( $n$ 22) received capsules containing primarily a blended fruit, vegetable and berry juice powder concentrate derived from the following: acerola cherry, apple, bilberry, blackberry, black currant, blueberry, beetroot, broccoli, cabbage, carrot, Concord grape, cranberry, elderberry, kale, orange, peach, papaya, parsley, pineapple, raspberry, red currant, spinach and tomato (Juice Plus $+{ }^{\circledR}$ 
Premium; NSA), as described previously ${ }^{(10)}$. Briefly, the FBV capsules provided $7.5 \mathrm{mg} \beta$-carotene, $200 \mathrm{mg}$ vitamin C, $60 \mathrm{mg}$ RRR- $\alpha$-tocopherol, $600 \mu \mathrm{g}$ folate and $63 \mathrm{~kJ} / \mathrm{d}$. Those subjects randomised to the placebo group ( $n$ 22) received identically appearing opaque white capsules containing microcrystalline cellulose. All subjects were instructed to take three capsules twice daily with meals, in agreement with the label use instructions for the retail product, for a total of six capsules per $\mathrm{d}$.

\section{Eligibility exercise test}

As part of eligibility testing, each subject performed an incremental exercise test on a treadmill ergometer (QUASARmed; HP Cosmos Sports \& Medical GmbH) to check the heart and circulatory function and for the determination of $\mathrm{VO}_{2 \max }$. A standard electrocardiogram was recorded throughout all exercise tests, which were supervised by a physician. Respiratory gas exchange variables were measured throughout the incremental exercise tests using a breath-by-breath mode (Metalyzer 3B; Cortex Biophysik GmbH).

\section{Endurance exercise test}

For the $30 \mathrm{~min}$ aerobic exercise tests, the walking speed was adjusted to $70 \%$ of individual $\mathrm{VO}_{2 \max }$ on the treadmill ergometer after the standardised breakfast described previously. All tests were performed on the same treadmill, with the same standardised room temperature $\left(20^{\circ} \mathrm{C}\right)$ and humidity (60\%). Blood pressure was measured at the beginning and every $10 \mathrm{~min}$ until the bout was completed.

\section{Blood collection and sample preparation}

At each laboratory visit, two EDTA blood samples were collected from each participant, in a supine position, from a medial cubital vein: before exercise (pre) and immediately post-exercise (post). This venous blood was collected to determine the concentrations of carbonyl proteins (CP), oxidised LDL (ox-LDL), total oxidation status of lipids (TOS), malondialdehyde (MDA), TNF- $\alpha$ and IL-6. After centrifugation for $10 \mathrm{~min}$, plasma was removed and frozen at $-70^{\circ} \mathrm{C}$ until analysis.

\section{Biochemical analyses}

CP concentration was quantified using ELISA methods developed previously by Buss \& Winterbourn ${ }^{(18)}$ and Alamdari et $a{ }^{(19)}$. These methods are based on the antibody recognition of carbonyl protein-bound 2,4-dinitrophenylhydrazine.

For ox-LDL determination, a commercially available immunosorbent kit (Mercodia AB) based on a direct sandwich technique was utilised.

The TOS assay determines total lipid peroxides (Immundiagnostik AG) by the detection of a coloured product from the reaction of a peroxidase with the peroxides in the sample, followed by the conversion of tetramethylbenzidine.

MDA concentration was determined according to a previously described HPLC method by Pilz et al. ${ }^{(20)}$ after derivatisation with 2,4-dinitrophenylhydrazine.
Both TNF- $\alpha$ and IL- 6 concentrations were analysed using commercially available ELISA kits with monoclonal antibodies (TNF- $\alpha$ : Immundiagnostik AG; IL-6: Invitrogen; LifeTech Austria).

\section{Microcirculation parameters}

Measurements were conducted in a supine position after a 10 min rest before and after the walking exercise bout, on the back of the hand, between the first and second metacarpal bone. This tissue photo spectrometry technology is also called 'oxygen to see' (Lea Instruments). All measurements were performed by the same technician. A laser Doppler effect, as described elsewhere ${ }^{(12,21)}$, was used to determine microcirculatory blood flow. For the determination of $\mathrm{O}_{2}$ saturation of $\mathrm{Hb}$ $\left(\mathrm{SO}_{2} \mathrm{Hb}\right)$ and relative $\mathrm{Hb}$ concentration ( $\mathrm{rHb}$ ), white-light tissue spectrometry was utilised: $\mathrm{SO}_{2} \mathrm{Hb}$ is identified by the colour of $\mathrm{Hb}$, as the degree of molecular $\mathrm{SO}_{2} \mathrm{Hb}$ relates to a certain colour. $\mathrm{rHb}$ was quantified using light absorption by the conversion of white light into red light, which is proportional to the concentration of $\mathrm{Hb}^{(22)}$. Blood flow and $\mathrm{rHb}$ are expressed in arbitrary units, whereas $\mathrm{SO}_{2} \mathrm{Hb}$ is expressed as a percentage of $\mathrm{O}_{2}$ on $\mathrm{Hb}$. This technology measures the microcirculation of blood from the skin surface to a $2 \mathrm{~mm}$ depth.

\section{Blood chemistry panel}

Standard blood chemistry was determined for eligibility testing after an overnight fast using EDTA plasma from the peripheral venous blood using a routine clinical chemistry analyser (Abbott Diagnostics).

\section{Statistical analyses and sample size calculation}

Per-protocol analyses were performed using IBM SPSS for Windows software, version 19.0 (SPSS Inc.). Data are presented as means and standard deviations. Data for pre-postcomparisons were adjusted for plasma volume changes as described elsewhere (except for $\mathrm{CP}$, as it is already expressed in relation to protein concentration $)^{(23)}$. Statistical significance was set at $P<0 \cdot 05$. The Shapiro-Wilk test was used to determine a normal distribution. Baseline characteristics, performance data, nutrient and clinical chemistry data were compared using the unpaired Student's $t$ test. Data obtained for CP, Ox-LDL, TOS, MDA, TNF- $\alpha$, IL-6, $\mathrm{SO}_{2} \mathrm{Hb}, \mathrm{rHb}$ and blood flow were analysed using a univariate, three-factorial, repeated-measures ANOVA. Factors were as follows: treatment (FBV or placebo); exercise (pre- and post-exercise); session (walking test 1 and walking test 2). Significant interactions and main effects were analysed by Bonferroni correction.

The sample size estimate of seventeen subjects per group was based on previous data on oxidation and inflammation markers (markers of primary outcome) and subjected to a probability of error $(\alpha=5 \%)$ and to a test power ( $1-\beta=80 \%$ ). Concerning the mean values, we assumed to discover a difference of $20 \%$ between the FBV and placebo groups after 8 weeks of treatment (and in comparison from pre- to post-exercise) and a standard deviation of $20 \%$ for the oxidation markers CP and MDA. For the mean values of 
TNF- $\alpha$ and IL-6, we assumed to discover a difference of $30 \%$ between the FBV and placebo groups after 8 weeks of treatment (and in comparison from pre- to post-exercise) and a standard deviation of $30 \%$. Allowing for an anticipated attrition of $20 \%$ in each group, twenty-two subjects per group were recruited to discover the assumed differences.

\section{Results}

\section{Study population and nutrition}

A CONSORT (consolidated standards of reporting trials) diagram outlining participant recruitment is depicted in Fig. 1.
Of the forty-four randomised women, forty-two completed the full programme and were included in the statistical analyses. There was one early termination in each study group: in the FBV group, one subject was disqualified at the follow-up visit due to weight loss $>3 \%$ of baseline body weight; in the placebo group, one person withdrew due to illness unrelated to the study.

The returned capsule count at the end of the study estimated a compliance $>85 \%$ in both groups. The groups did not differ in age, BMI, $\mathrm{VO}_{2 \max }, \mathrm{VO}_{2 \max }$ related to body weight, maximum workload $\left(P_{\max }\right)$, clinical blood chemistry variables and habitual diet $(P>0 \cdot 05$; Table 1$)$.

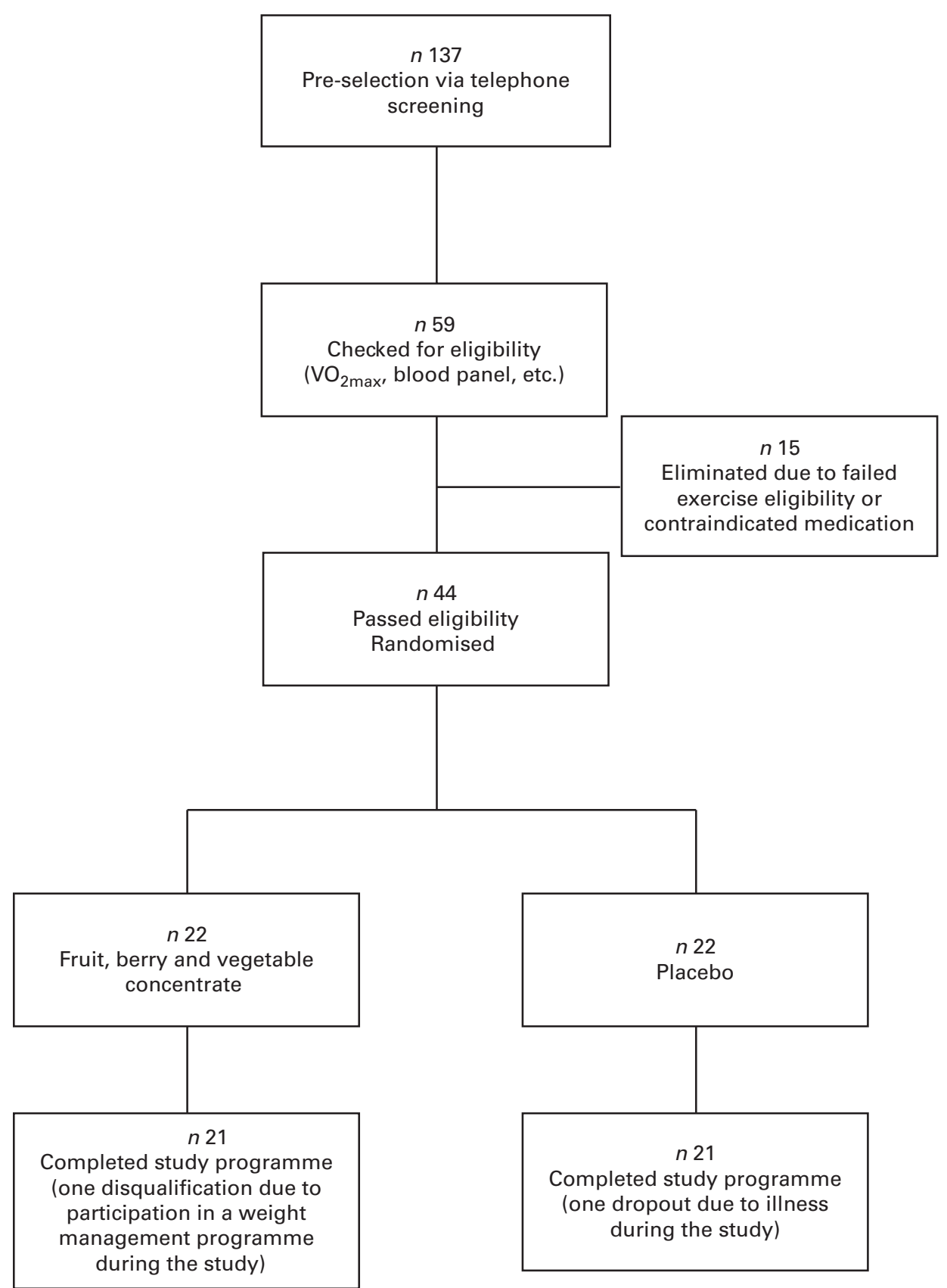

Fig. 1. CONSORT (consolidated standards of reporting trials) diagram. 
Table 1. Baseline characteristics, performance data, clinical chemistry and nutrition data of the forty-two pre-menopausal, obese, but otherwise healthy, women

(Mean values and standard deviations)

\begin{tabular}{|c|c|c|c|c|c|}
\hline \multirow[b]{2}{*}{ Variables } & \multirow[b]{2}{*}{ Reference values ${ }^{*} \dagger$} & \multicolumn{2}{|c|}{ FBV $(n 21)$} & \multicolumn{2}{|c|}{ Placebo ( $n 21)$} \\
\hline & & Mean & SD & Mean & SD \\
\hline Age (years) & & $40 \cdot 8$ & 3.7 & $41 \cdot 3$ & $4 \cdot 2$ \\
\hline BMI $\left(\mathrm{kg} / \mathrm{m}^{2}\right)$ & & $34 \cdot 3$ & $4 \cdot 2$ & $34 \cdot 6$ & 3.5 \\
\hline Weight (kg) & & $92 \cdot 2$ & $12 \cdot 9$ & $91 \cdot 6$ & $11 \cdot 3$ \\
\hline $\mathrm{VO}_{2 \max }(\mathrm{ml})$ & & 2198 & 127 & 2259 & 169 \\
\hline $\mathrm{VO}_{2 \max }(\mathrm{ml} / \mathrm{kg}$ per min $)$ & & $25 \cdot 8$ & 3.5 & $27 \cdot 1$ & $3 \cdot 6$ \\
\hline$P_{\max }(\mathrm{km} / \mathrm{h})+$ slope $(\%)$ & & $6+7$ & 1.87 & $6+7$ & 1.69 \\
\hline$P_{68 \% \mathrm{VO}_{2 \max }}(\mathrm{km} / \mathrm{h})$ & & 6 & 0.62 & 6 & 0.55 \\
\hline \multicolumn{6}{|l|}{ Clinical chemistry } \\
\hline Glucose $(\mathrm{mmol} / \mathrm{l})$ & $3 \cdot 9-6 \cdot 1$ & $5 \cdot 1$ & 1.5 & $5 \cdot 0$ & 1.4 \\
\hline $\mathrm{Hb}(\mathrm{g} / \mathrm{l})$ & $115-155$ & 126 & 13 & 130 & 16 \\
\hline $\mathrm{Fe}(\mu \mathrm{mol} / \mathrm{l})$ & $11-29$ & $16 \cdot 4$ & 5.5 & $17 \cdot 6$ & 5.9 \\
\hline Ferritin $(\mu \mathrm{g} / \mathrm{l})$ & $18-300$ & $78 \cdot 4$ & 55.5 & 89.4 & $59 \cdot 1$ \\
\hline Cholesterol $(\mathrm{mmol} / \mathrm{l})$ & $<6.35$ & 5.47 & 1.73 & 5.56 & 1.23 \\
\hline $\mathrm{HDL}(\mathrm{mmol} / \mathrm{l})$ & $0.80-2 \cdot 35$ & $1 \cdot 27$ & 0.28 & $1 \cdot 11$ & 0.43 \\
\hline TAG (mmol/l) & $<1.80$ & 1.27 & 0.62 & 1.41 & 0.56 \\
\hline \multicolumn{6}{|c|}{ Average diet/d from the $7 \mathrm{~d}$ diet record } \\
\hline Energy (kJ/d) & 7927 & 7745 & 454 & 7923 & 392 \\
\hline Fat & $<30 \%$ of $\mathrm{kJ} / \mathrm{d}$ & $38.5 \%$ & $7 \cdot 2$ & $38.9 \%$ & $8 \cdot 1$ \\
\hline Protein & $10-15 \%$ of $\mathrm{kJ} / \mathrm{d}$ & $15 \cdot 7 \%$ & $2 \cdot 1$ & $16 \cdot 3 \%$ & 3.2 \\
\hline Carbohydrates & $>50 \%$ of $\mathrm{kJ} / \mathrm{d}$ & $43.9 \%$ & $9 \cdot 1$ & $43 \cdot 2 \%$ & $10 \cdot 3$ \\
\hline Alcohol (\%) & $<3.5$ & 1.9 & $1 \cdot 2$ & 1.5 & 0.9 \\
\hline Water (ml) & 2600 & 2162 & 95 & 2022 & 95 \\
\hline Fibre $(\mathrm{g})$ & 30 & 21 & 7 & 19 & 6 \\
\hline$\beta$-Carotene $(\mathrm{mg})$ & $4 \cdot 8$ & 0.14 & 0.09 & $0 \cdot 17$ & 0.11 \\
\hline Fruit and vegetable portions/d & 5 & $1-2$ & & $1-2$ & \\
\hline
\end{tabular}

FBV, fruit, berry and vegetable juice powder concentrate; $P_{\max }$, maximal performance; $P_{68 \% \text { vo2max }}$, performance $68 \%$ of maximal oxygen uptake. ${ }^{*}$ Reference intervals and upper limits for clinical chemistry parameters ${ }^{(54)}$.

$\dagger$ Reference values for dietary intake (RDA) in Germany, Austria and Switzerland ${ }^{(55)}$

\section{0 min controlled exercise bout}

The post-exercise analyses revealed that these women performed at 68.2 (SD $3 \cdot 1) \%$ of individual $\mathrm{VO}_{2 \max }$. The average walking performance was approximately $6 \mathrm{~km} / \mathrm{h}$ (Table 1 ). There were no significant differences between the FBV and placebo groups for these parameters $(P>0.05)$.

\section{Carbonyl proteins}

The mean values of both groups were comparable with healthy people of this age (reference interval $0.37-1.16 \mathrm{nmol} / \mathrm{mg}$ ). There were no differences between the groups at baseline, pre- and post-exercise. After 8 weeks of supplementation, there was a significant difference between the FBV and placebo groups ( $P_{\mathrm{Tx}}=0.022 ;$ Fig. 2$)$, both pre- and post-exercise. The FBV group had significantly lower $\mathrm{CP}$ concentrations compared with the placebo group. The model of exercise had no influence on $\mathrm{CP}$ concentrations.

\section{Oxidised $L D L$}

There were no differences between the groups at baseline, but a significant difference after 8 weeks of FBV or placebo supplementation $\left(P_{\mathrm{Tx}}=0.015 ;\right.$ Fig. 3$)$. The FBV group showed lower concentrations compared with the placebo group. The model of exercise had no influence on ox-LDL concentrations. However, all concentrations were within the reference interval, at the beginning and end of the study, pre- and post-exercise (30-80 U/1).

\section{Total oxidation status}

There were no differences between the groups at baseline, preand post-exercise. After 8 weeks of supplementation, there was a significant difference between the groups $\left(P_{\mathrm{Tx}}=0 \cdot 010 ;\right.$ Fig. 4$)$. After the supplementation period, FBV supplementation had reduced the elevated baseline values (reference cut-off $<350 \mu \mathrm{M}-\mathrm{H}_{2} \mathrm{O}_{2}$ ) from $>900 \mu \mathrm{m}-\mathrm{H}_{2} \mathrm{O}_{2}$ down to approximately $750 \mu \mathrm{M}-\mathrm{H}_{2} \mathrm{O}_{2}$, which is still above the reference interval. The model of exercise had no influence on TOS.

\section{Malondialdehyde}

There were no differences between the groups at baseline and after 8 weeks of supplementation, pre- and post-exercise, with all concentrations within the reference interval (2.16 (SD $0 \cdot 29) \mathrm{nmol} / \mathrm{ml}$; data not shown). Also, the model of exercise had no influence on MDA concentrations.

\section{$T N F-\alpha$}

Despite the typically high standard deviation for TNF- $\alpha$, due to the established cytokine inter-individual variability, the data were normally distributed. There were no differences between the groups at baseline, although pre- and post-exercise 


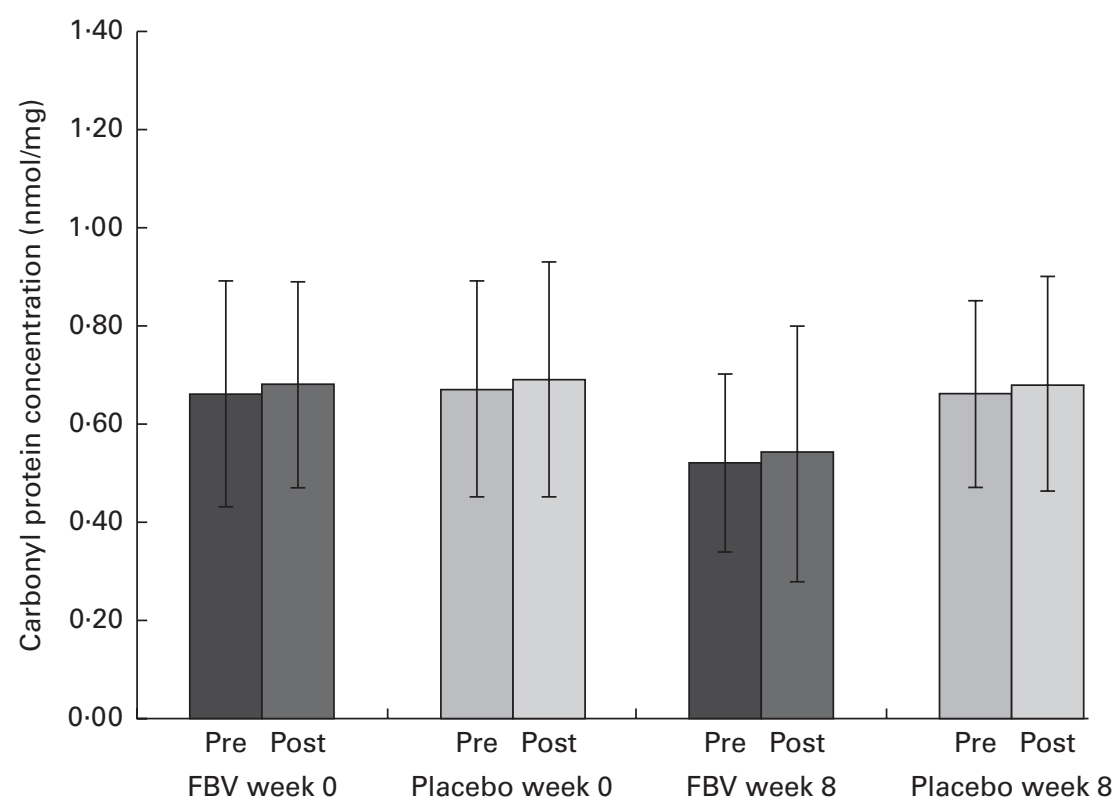

Fig. 2. Plasma concentrations of carbonyl groups bound on protein in overweight and obese women ( $n$ 42) before and after 8 weeks of supplementation, and preand post-30 min of walking exercise. Values are means ( $n 21$ per group), with standard deviations represented by vertical bars. There was a significant effect of treatment after 8 weeks, with no influence of exercise $(P=0.022$; ANOVA). FBV, fruit, berry and vegetable concentrate group.

concentrations at baseline (mean value $>24 \mathrm{pg} / \mathrm{ml}$ ) exceeded the upper reference limit $(<20 \mathrm{pg} / \mathrm{ml}$; Fig. 5). Following 8 weeks of FBV supplementation, pre- and post-exercise, TNF- $\alpha$ concentrations were within the normal physiological range, whereas the values remained elevated in the placebo group. Hence, there was a significant difference between the FBV and placebo groups after 8 weeks of intervention. The model of exercise had no influence on TNF- $\alpha$.

\section{IL-6}

There were no observed differences between the groups at baseline and after 8 weeks of capsule supplementation, both pre- and post-exercise. Also, the model of exercise had no influence. IL-6 concentrations for all subjects remained below the reference cut-off value $(<11.3 \mathrm{pg} / \mathrm{ml}$; data not shown) throughout the investigation.

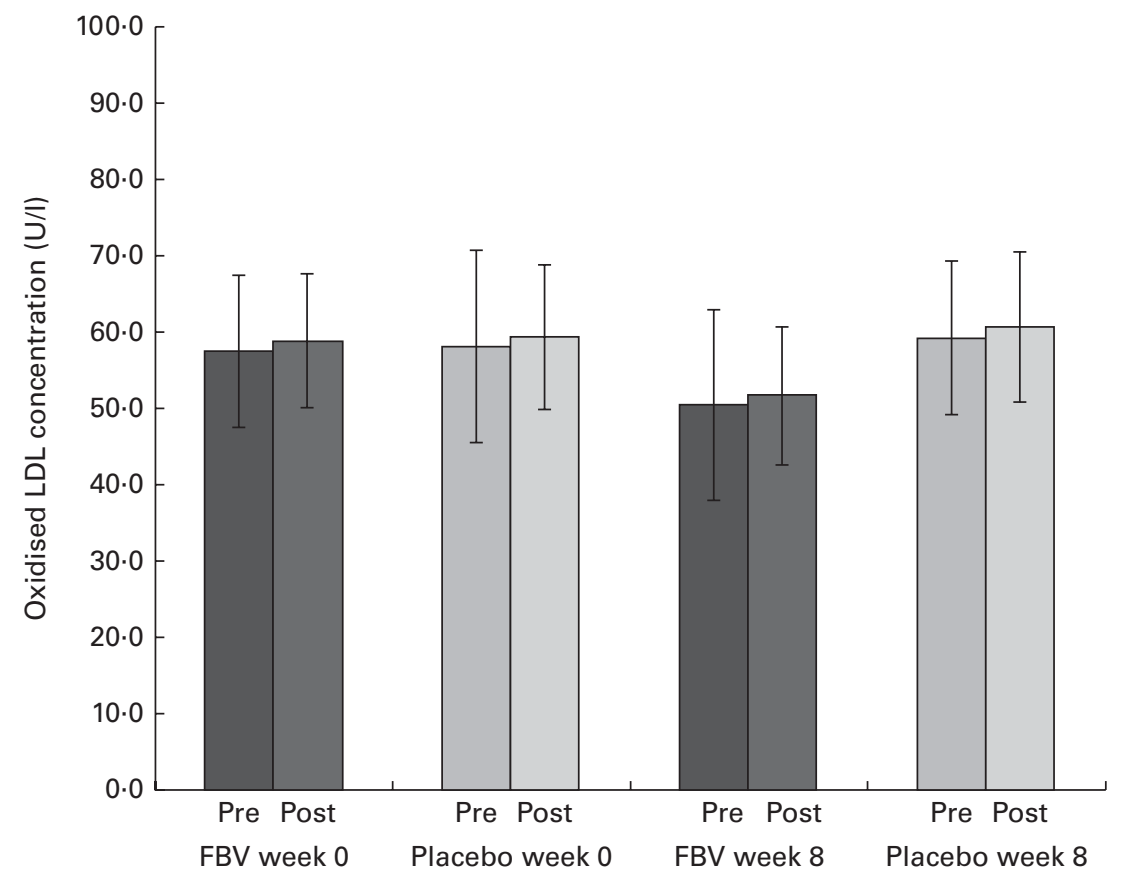

Fig. 3. Plasma oxidised LDL concentrations in overweight and obese women ( $n$ 42) before and after 8 weeks of supplementation, and pre- and post-30 min of walking exercise. Values are means ( $n 21$ per group), with standard deviations represented by vertical bars. There was a significant effect of treatment after 8 weeks, with no influence of exercise $(P=0.015$; ANOVA). FBV, fruit, berry and vegetable concentrate group. 


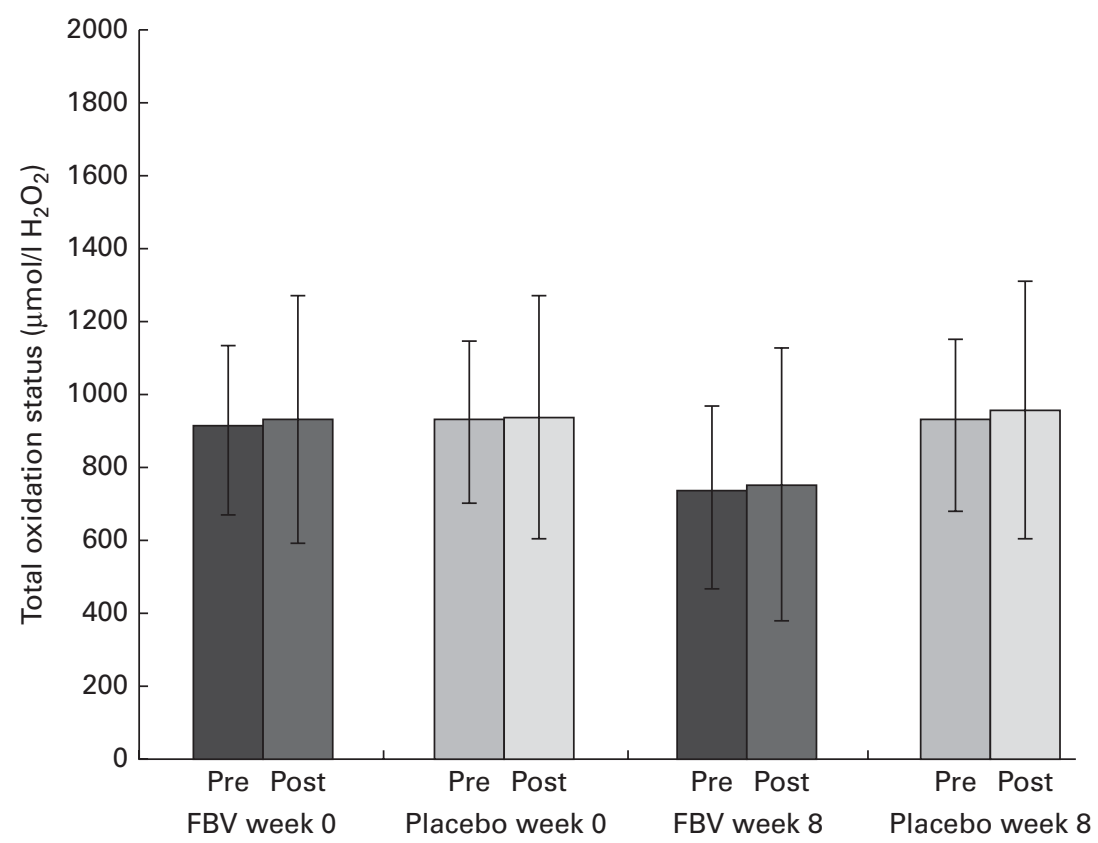

Fig. 4. Plasma total oxidation status of lipids in overweight and obese women $(n 42)$ before and after 8 weeks of supplementation, and pre- and post-30 min of walking exercise. Values are means ( $n 21$ per group), with standard deviations represented by vertical bars. There was a significant effect of treatment after 8 weeks, with no influence of exercise $(P=0.010$; ANOVA). FBV, fruit, berry and vegetable concentrate group.

\section{Skin microcirculation}

All values were within the normal limits provided by the equipment manufacturer for healthy people. There were no significant differences between the groups at baseline with pre- and post-exercise blood flow. After the 8-week supplement period, blood flow was significantly higher in the FBV group

compared with placebo $\left(P_{\mathrm{Tx}}=0 \cdot 029\right)$. There was also a significant increase due to exercise from pre- to post-exercise $(P=0.004)$ in both groups, at baseline and after 8 weeks.

There were no differences in $\mathrm{SO}_{2} \mathrm{Hb}$ between the groups at baseline, pre- and post-exercise. Following the 8-week

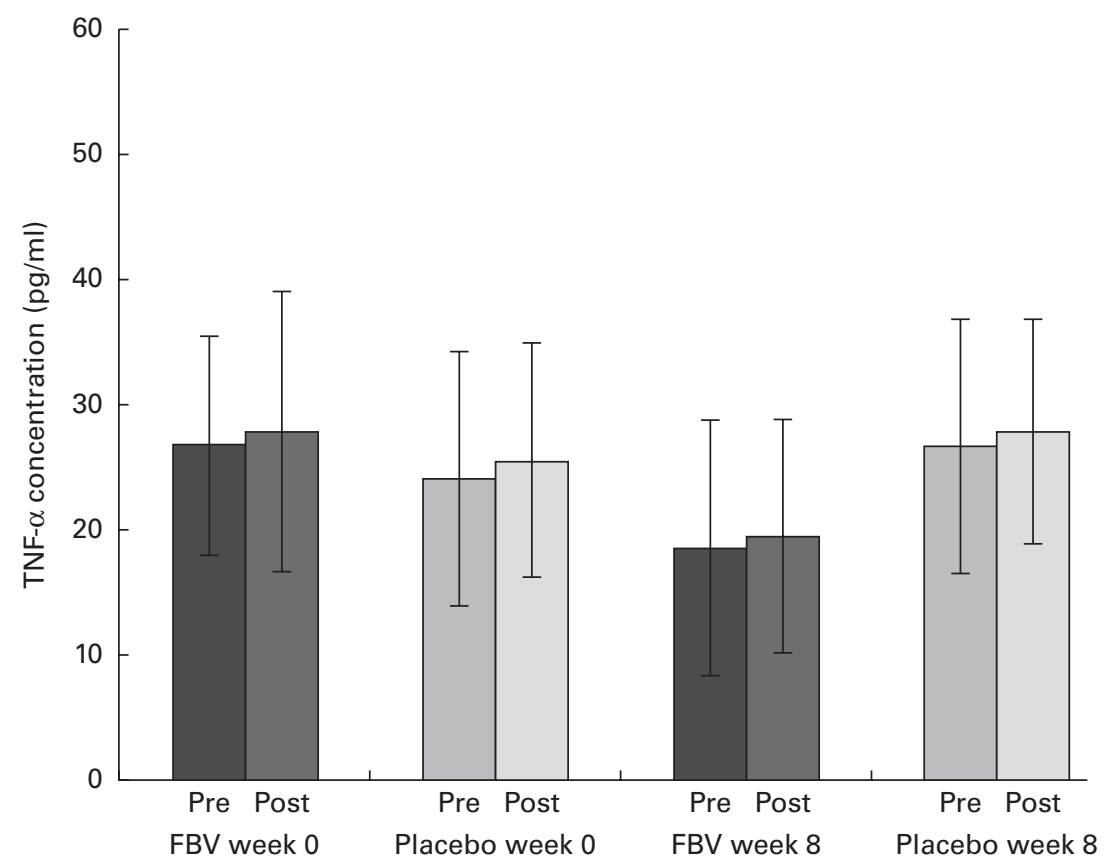

Fig. 5. Plasma TNF- $\alpha$ concentrations in overweight and obese women ( $n$ 42) before and after 8 weeks of treatment, and pre- and post-30 min of walking exercise. Values are means ( $n 21$ per group), with standard deviations represented by vertical bars. There was a significant effect of treatment after 8 weeks, with no influence of exercise $(P=0.011$; ANOVA). FBV, fruit, berry and vegetable concentrate group. 
capsule supplement period, $\mathrm{SO}_{2} \mathrm{Hb}$ was significantly higher in the FBV group compared with placebo $\left(P_{\mathrm{Tx}}=0.032\right)$, pre- and post-exercise (Table 2$)$. The model of exercise also affected the $\mathrm{SO}_{2} \mathrm{Hb}$ values, with increased values from pre- to postexercise in both groups at both time points, but this effect did not reach significance $\left(P_{\mathrm{Ex}}=0.075\right)$.

As with $\mathrm{SO}_{2} \mathrm{Hb}$, there were no differences between the groups at baseline with pre- and post-exercise for $\mathrm{rHb}$. After the 8-week supplement period, $\mathrm{rHb}$ was significantly higher in the FBV group compared with placebo $\left(P_{\mathrm{Tx}}=0.041\right)$, pre- and postexercise (Table 2). There was also a significant effect of exercise

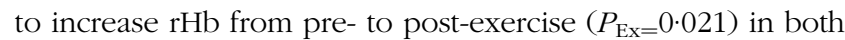
groups, at baseline and after 8 weeks of treatment.

\section{Discussion}

Increased protein and lipid oxidation, as well as low-grade inflammation, are conditions associated with increased cardiovascular and chronic disease risk ${ }^{(1,2,5)}$. Obese people are at a higher risk of developing chronic medical conditions, thus making an interesting study population. The present investigation was primarily focused on the responses of oxidation, inflammation and capillary microcirculation markers in obese pre-menopausal women, after an 8-week intervention with FBV or placebo capsules, followed by a single bout of aerobic exercise. The resulting data show, after the 8-week study period, (1) compared with placebo, the FBV group had a reduction in the markers of protein oxidation, ox-LDL and total lipid oxidation, and lower concentrations of the chronic inflammation marker TNF- $\alpha$. The study also revealed that (2) FBV supplementation increased the microcirculation in the skin. (3) A 30 min walking exercise at $70 \%$ of $\mathrm{VO}_{2 \max }$ increased blood flow and $\mathrm{rHb}$ in the skin in both groups (secondary outcome) and (4) the walking exercise did not generate additional oxidative stress in these sedentary obese women (tertiary outcome).

\section{Oxidative stress markers}

In the present study, protein oxidation (as assessed by $\mathrm{CP}$ ) was decreased after the 8-week FBV supplementation period. The decrease, or attenuation, of $\mathrm{CP}$ in healthy and trained subjects in response to this FBV supplementation has been reported previously $^{(10,24-26)}$, and this effect is also now demonstrated in these obese women. The bioavailability of FBV antioxidant vitamins and phytonutrients has been reported previously ${ }^{(11,27,28)}$ and may be the explanation for the consistent observation of decreased or attenuated protein oxidation. However, all CP values remained within the reference interval throughout the investigation in all participants.

Similar to CP, ox-LDL was reduced after 8 weeks of FBV supplementation. Ox-LDL is one of the few recognised parameters of the European Food Safety Authority to estimate oxidative damage to lipids ${ }^{(29)}$. It is also a recognised cardiovascular risk factor associated with obesity ${ }^{(30-34)}$. Over the study period, the FBV group had a $12 \%$ reduction in ox-LDL concentrations (from 58 to $51 \mathrm{U} / 1$ ). A similar reduction has been reported in heavy smokers using the same FBV capsules for a 3-month 
period $^{(35)}$. This consistent finding may be due to the antioxidant activity of FBV, protecting LDL from oxidation.

TOS, another marker of lipid peroxidation, was elevated at all measured time points, indicating higher concentrations of total lipid peroxides in this cohort of obese women. This surrogate marker is a comprehensive indicator of lipid peroxidation, and thus not as specific for the oxidation of certain molecules, unlike ox-LDL or MDA. The elevated TOS values observed here might reflect a higher oxidation state of MUFA or oxidation derived from other sources, such as advanced glycation end products. These substances originate from a fat-rich diet ${ }^{(36)}$, which was consumed by the women in the present study (Table 1). The antioxidant functions of FBV decreased TOS concentrations; however, the values remained elevated at the end of the present 8-week study. It would be interesting to observe the response of this marker after long-term FBV supplementation.

MDA is a commonly used marker to estimate lipid peroxidation $^{(37-39)}$. It is an indicator of damage to PUFA ${ }^{(39)}$. Protein-bound MDA was assessed in the present investigation. Neither the capsule treatment nor exercise was distinctive enough to effect changes in MDA concentrations, which remained within the reference interval throughout the study.

\section{Inflammatory markers}

Low-grade chronic systemic inflammation has commonly been reported in obese populations, which is accompanied by increased systemic levels of cytokines including TNF- $\alpha$ and IL-6 ${ }^{(14,27)}$

The changes in TNF- $\alpha$ concentrations in the FBV group were remarkable. At baseline, both study groups had elevated concentrations, exceeding the reference cut-off value $(20 \mathrm{pg} / \mathrm{ml})$. After 8 weeks of supplementation, TNF- $\alpha$ in the FBV group was within the reference limit. This is an important finding in the context of the frequently postulated involvement of TNF- $\alpha$ in obesity and imbalanced insulin metabolism ${ }^{(40-42)}$. It has been postulated that adipose tissue, which produces TNF- $\alpha$, is the main source of circulating TNF- $\alpha^{(43,44)}$. However, it has also been observed that a low intake of $\beta$-carotene, found in fruit and vegetables, is inversely related to $\operatorname{TNF}-\alpha$, due to a diet-dependent decreased antioxidant and anti-inflammatory capacity $^{(9)}$. In the present study, overweight and obese women had increased TNF- $\alpha$ concentrations at baseline, along with suboptimal dietary intakes of $\beta$-carotene and fruit and vegetables (Table 1 ). We are aware that the lack of serum $\beta$-carotene measurements in the present study is a limitation. On the other hand, although $\beta$-carotene was not measured, others have consistently reported increased $\beta$-carotene concentrations in studies using the same FBV supplementation ${ }^{(27,28,45)}$. Therefore, it is reasonable to expect the FBV group in the present study also had increased $\beta$-carotene concentrations, which may have contributed to the decrease observed in TNF- $\alpha$ in the FBV group, particularly since body weight and exercise habits remained constant during the study.

All subjects had IL-6 values within the normal limit and no changes were observed during the study. Other studies have also reported that FBV supplementation did not influence
IL-6 concentrations ${ }^{(10,11)}$, and obviously, the model of exercise was not exhaustive enough to generate IL-6 from muscle inflammation.

\section{Microcirculation markers}

The maintenance of microvascular integrity is related to intact endothelial NO metabolism and protective against adipositylinked CVD ${ }^{(46,47)}$. As NO metabolism is also dependent on redox biochemistry, we hypothesised that FBV supplementation might exert the effects on the microcirculation assessed on the easily accessible skin surface.

All skin microcirculation values were within the reference limit at all the time points assessed; however, these values did increase with both FBV supplementation and exercise. The observed increases in blood flow, $\mathrm{SO}_{2} \mathrm{Hb}$ and $\mathrm{rHB}$ indicate reduced $\mathrm{O}_{2}$ extraction and vasodilation of the blood vessels. In theory, perhaps the constituents of FBV would have stimulated NO metabolism, for better oxygenation in the capillaries near the skin surface. Plotnick et al. $^{(48)}$ demonstrated that this FBV provides dietary nitrate, resulting in an increase in nitrate/nitrite levels after a 4-week study of FBV supplementation in healthy volunteers, accompanied by improved flowmediated dilatation after a high-fat meal. Once NO is generated via nitrate and nitrite reductase, it reduces $\mathrm{O}_{2}$ extraction from $\mathrm{Hb}$ and also the $\mathrm{O}_{2}$ cost in the tissue ${ }^{(49)}$. In addition to the nutrients from FBV, it is presumable that exercise affected NO generation via increased blood flow, exerting shear stress on endothelial cell membranes. It has been well established that an increase in blood flow stimulates vascular endothelial cells and promotes the production of various vasodilator substances including NO or prostacyclin ${ }^{(50-52)}$.

\section{Effect of exercise on oxidation}

A null effect was observed in these women with regard to exercise-generated oxidation. The $30 \mathrm{~min}$ exercise bout at close to $70 \%$ of $\mathrm{VO}_{2 \max }$ did not result in increased protein and lipid oxidation (indicated here via CP, Ox-LDL, TOS and MDA). This is in line with the recent findings that demonstrated a lack in the increase of $\mathrm{CP}$ concentrations with $70 \%$ $\mathrm{VO}_{2 \max }$ exercise for $40 \mathrm{~min}$ in trained men ${ }^{(53)}$. However, to our knowledge, the present investigation demonstrated for the first time that obese, but otherwise healthy, women can perform walking exercise at $70 \% \mathrm{VO}_{2 \max }$ over $30 \mathrm{~min}$ without generating oxidative stress. This suggests that exercise regimens for basically healthy obese women should also include aerobic exercise of higher intensity than is usually applied to accelerate improvements in energy consumption capacity and aerobic fitness.

\section{Conclusions}

The overall result from the present study is that 8 weeks of supplementation with an encapsulated fruit, berry and vegetable concentrate decreased the oxidation of proteins and lipids in plasma and reduced low-grade inflammation in overweight and obese women compared with the placebo group. 
Further, FBV supplementation and 30 min of aerobic walking exercise complemented each other to promote the microcirculation in the skin. In addition, we demonstrated that this population does not suffer oxidative damage or inflammation after $70 \% \mathrm{VO}_{2 \max }$ walking intensity over $30 \mathrm{~min}$.

\section{Acknowledgements}

The present study was funded by a competitive research grant from NSA LLC (Collierville, Tennessee, USA) to the Institute of Nutrient Research and Sport Nutrition (Graz, Austria). The authors wish to thank Dr Anita M. Boddie for improving the English of the manuscript.

M. L. was the principal investigator and contributed to the development of the overall research plan and study protocol, project management and study oversight, statistical analyses and the preparation of the manuscript; G. O. contributed to the microcirculation measurements, data collection, statistical analyses and manuscript revision; K. S. was involved in the performance diagnostics, data collection and manuscript revision; G. C. participated in the blood sampling, laboratory logistics, data collection and manuscript revision; L. H., G. L. and J. F. G. contributed to the laboratory analyses, data collection and manuscript revision; $\mathrm{S}$. H. was responsible for the laboratory analyses, data collection and the preparation of the manuscript.

M. L. and G. O. are affiliated with the Institute of Nutrient Research and Sport Nutrition. The rest of the authors declare no conflict of interest.

\section{References}

1. Bakker GCM, vanErk MJ, Pellis L, et al. (2010) An antiinflammatory dietary mix modulates inflammation and oxidative and metabolic stress in overweight men: a nutrigenomics approach. Am J Clin Nutr 91, 1044-1059.

2. Fujita K, Nishizawa H, Funahashi T, et al. (2006) Systemic oxidative stress is associated with visceral fat accumulation and the metabolic syndrome. Circ J 70, 1437-1442.

3. Kraemer-Aguiar LG, Laflor CM \& Bouskela E (2008) Skin microcirculatory dysfunction is already present in normoglycemic subjects with metabolic syndrome. Metabolism 57, 1740-1746.

4. Fain JN (2006) Release of interleukins and other inflammatory cytokines by human adipose tissue is enhanced in obesity and primarily due to the nonfat cells. Vitam Horm $\mathbf{7 4}$, 443-477.

5. Smith DT, Carr LJ, Dorozynski C, et al. (2009) Internetdelivered lifestyle physical activity intervention: limited inflammation and antioxidant capacity efficacy in overweight adults. J Appl Physiol 106, 49-56.

6. Francischetti EA, Tibirica E, da Silva EG, et al. (2001) Skin capillary density and microvascular reactivity in obese subjects with and without metabolic syndrome. Microvasc Res 81, 325-330.

7. Dragsted LO, Pedersen A, Hermetter A, et al. (2004) The 6-a-day study: effects of fruit and vegetables on markers of oxidative stress and antioxidative defense in healthy smokers. Am J Clin Nutr 79, 1060-1072.

8. Cao G, Booth SL, Sadowski JA, et al. (1998) Increases in human plasma antioxidant capacity after consumption of controlled diets high in fruit and vegetables. Am J Clin Nutr 68, 1081-1087.

9. Holt EM, Steffen LM, Moran A, et al. (2009) Fruit and vegetable consumption and its relation to markers of inflammation and oxidative stress in adolescents. J Am Diet Assoc 109, 414-421.

10. Lamprecht M, Oettl K, Schwaberger G, et al. (2007) Several indicators of oxidative stress, immunity, and illness improved in trained men consuming an encapsulated juice powder concentrate for 28 weeks. J Nutr 137, 2737-2741.

11. Nantz MP, Rowe CA, Nieves C Jr, et al. (2006) Immunity and antioxidant capacity in humans is enhanced by consumption of a dried, encapsulated fruit and vegetable concentrate. J Nutr 136, 2606-2610.

12. DeSpirt S, Sies H, Tronnier H, et al. (2012) An encapsulated fruit and vegetable juice concentrate increases skin microcirculation in healthy women. Skin Pharmacol Physiol 25, 2-8.

13. Radak Z, Taylor AW, Ohno H, et al. (2001) Adaptation to exercise-induced oxidative stress: from muscle to brain. Exerc Immunol Rev 7, 90-107.

14. Petersen AMW \& Pedersen BK (2005) The anti-inflammatory effect of exercise. J Appl Physiol 98, 1154-1162.

15. Pasqualini L, Schillaci G, Innocente S, et al. (2010) Lifestyle intervention improves microvascular reactivity and increases serum adiponectin in overweight hypertensive patients. Nutr Metab Cardiovasc Dis 20, 87-92.

16. Tew GA, Saxton JM \& Hodges GJ (2012) Exercise training and the control of skin blood flow in older adults. $J$ Nutr Health Aging 16, 237-241.

17. Expertenkommission der DGSP - Deutsche Gesellschaft für Sportmedizin und Prävention (Commission of Experts of the German Society of Sports Medicine and Prevention) (2002) Leitlinien zur Belastungsuntersuchung in der Sportmedizin. Ebene IV - 03/2002 (Guidelines for Testing in Sports Medicine. Level IV - March 2002). Germany: Commission of Experts of the German Society of Sports Medicine and Prevention.

18. Buss IH \& Winterbourn CC (2002) Protein carbonyl measurement by ELISA. Methods Mol Biol 186, 123-128.

19. Alamdari DH, Kostidou E, Palets K, et al. (2005) High sensitivity enzyme-linked immunosorbent assay (ELISA) method for measuring protein carbonyl in samples with low amounts of protein. Free Radic Biol Med 39, 1362-1367.

20. Pilz J, Meinekea I \& Gleitera CH (2000) Measurement of free and bound malondialdehyde in plasma by high-performance liquid chromatography as the 2,4-dinitrophenyl-hydrazine derivative. J Chromatogr B Biomed Sci Appl 742, 315-325.

21. Stirban A, Nandrean S, Götting C, et al. (2010) Effects of $n-3$ fatty acids on macro- and microvascular function in subjects with type 2 diabetes mellitus. Am J Clin Nutr 91, 808-813.

22. Krug A (2006) Mikrozirkulation und Sauerstoffversorgung des Gewebes - Methode des sogenannten O2C (oxygen to see) (German) (Microcirculation and oxygen supply to tissue - the O2C (oxygen to see) method). Phlebologie 36, $300-312$.

23. Dill DB \& Costill DL (1999) Calculation of percentage changes in volumes of blood, plasma, and red cells in dehydration. J Appl Physiol 73, 1265-1272.

24. Lamprecht M, Oettl K, Schwaberger G, et al. (2009) Protein modification responds to exercise intensity and antioxidant supplementation. Med Sci Sports Exerc 41, 155-163.

25. Goldfarb AH, Garten RS, Cho C, et al. (2011) Effects of a fruit/berry/vegetable supplement on muscle function and oxidative stress. Med Sci Sports Exerc 43, 501-508.

26. Bloomer RJ, Goldfarb AH \& McKenzie MJ (2006) Oxidative stress response to aerobic exercise: comparison of antioxidant supplements. Med Sci Sports Exerc 38, 1098-1105. 
27. Canas JA, Damaso L, Altomare A, et al. (2012) Insulin resistance and adiposity in relation to serum $\beta$-carotene levels. J Pediatr 161, 58-64.

28. Kawashima A, Madarame T, Koike H, et al. (2007) Four week supplementation with mixed fruit and vegetable juice concentrates increased protective serum antioxidants and folate and decreased plasma homocysteine in Japanese subjects. Asia Pac J Clin Nutr 16, 411-421.

29. European Food Safety Authority; EFSA Panel on Dietetic Products, Nutrition and Allergies (NDA) (2011) Guidance on the scientific requirements for health claims related to antioxidants, oxidative damage and cardiovascular health. EFSA $J$ 9, 2474 .

30. Linna MS, Borg P, Kukkonen-Harjula K, et al. (2007) Successful weight maintenance preserves lower levels of oxidized LDL achieved by weight reduction in obese men. Int $J$ Obes (Lond) 31, 245-253.

31. Meisinger C, Baumert J, Khuseyinova N, et al. (2005) Plasma oxidized low-density lipoprotein, a strong predictor for acute coronary heart disease events in apparently healthy, middle-aged men from the general population. Circulation 112, 651-657.

32. Njajou OT, Kanaya AM, Holvoet P, et al. (2009) Association between oxidized LDL, obesity and type 2 diabetes in a population-based cohort, the health aging and body composition study. Diabetes Metab Res Rev 25, 733-739.

33. Vasankari T, Fogelholm M, Kukkonen-Harjula K, et al. (2001) Reduced oxidized low-density lipoprotein after weight reduction in obese premenopausal women. Int $J$ Obes Relat Metab Disord 25, 205-211.

34. Vasankari TJ, Kujala UM, Vasankari TM, et al. (1998) Reduced oxidized LDL levels after a 10-month exercise program. Med Sci Sports Exerc 30, 1496-1501.

35. Novembrino C, Cighetti G, DeGiuseppe R, et al. (2011) Effects of encapsulated fruit and vegetable juice powder concentrates on oxidative status in heavy smokers. $J \mathrm{Am}$ Coll Nutr 30, 49-56.

36. Tomino Y, Hagiwara S \& Gohda T (2011) AGE-RAGE interaction and oxidative stress in obesity-related renal dysfunction. Kidney Int 80, 133-135.

37. Deepa D, Jayakumari B \& Thomas SV (2008) Lipid peroxidation in women with epilepsy. Ann Indian Acad Neurol 11, 44-46.

38. Lamprecht M, Hofmann P, Greilberger JF, et al. (2009) Increased lipid peroxidation in trained men after 2 weeks of antioxidant supplementation. Int J Sport Nutr Exerc Metab 19, 385-399.

39. Esterbauer H, Schaur RJ \& Zollner H (1991) Chemistry and biochemistry of 4-hydroxynonenal, malondialdehyde and related aldehydes. Free Radic Biol Med 11, 81-128.

40. Hotamisligil GS (1999) Mechanisms of TNF-alpha-induced insulin resistance. Exp Clin Endocrinol Diabetes 107, 119-125.

41. Hotamisligil GS, Peraldi P, Budavari A, et al. (1996) IRS-1mediated inhibition of insulin receptor tyrosine kinase activity in TNF-alpha- and obesity-induced insulin resistance. Science 271, 665-668.

42. Bruce CR \& Dyck DJ (2004) Cytokine regulation of skeletal muscle fatty acid metabolism: effect of interleukin- 6 and tumor necrosis factor- $\alpha$. Am J Physiol Endocrinol Metab 287, E616-E621.

43. Hotamisligil GS, Shargill NS \& Spiegelman BM (1993) Adipose expression of tumor necrosis factor-alpha: direct role in obesity-linked insulin resistance. Science 259, 87-91.

44. Coppack SW (2001) Pro-inflammatory cytokines and adipose tissue. Proc Nutr Soc 60, 349-356.

45. Jin Y, Cui X, Singh U, et al. (2010) Systematic inflammatory load in humans is suppressed by consumption of two formulations of dried, encapsulated juice concentrate. Mol Nutr Food Res 54, 1506-1514.

46. Clerk LH, Vincent MA, Hahn LA, et al. (2006) Obesity blunts insulin-mediated microvascular recruitment in human forearm muscle. Diabetes 55, 1436-1442.

47. Jonk AM, Houben AJ, DeJongh RT, et al. (2007) Microvascular dysfunction in obesity: a potential mechanism in the pathogenesis of obesity-associated insulin resistance and hypertension. Physiology (Bethesda) 22, 252-260.

48. Plotnick GD, Corretti MC, Vogel RA, et al. (2003) Effect of supplemental phytonutrients on impairment of the flowmediated brachial artery vasoactivity after a single high-fat meal. J Am Coll Cardiol 41, 1744-1749.

49. Bailey SJ, Winyard P, Vanhatalo A, et al. (2009) Dietary nitrate supplementation reduces the $\mathrm{O}_{2}$ cost of low-intensity exercise and enhances tolerance to high-intensity exercise in humans. J Appl Physiol 107, 1144-1155.

50. Jungersten L, Ambring A, Wall B, et al. (1997) Both physical fitness and acute exercise regulate nitric oxide formation in healthy humans. $J$ Appl Physiol 82, 760-764.

51. Matsumoto A, Hirai Y, Momomura S, et al. (1994) Increased nitric oxide production during exercise. Lancet 343, $849-850$

52. Roberts CK, Barnard RJ, Jasman A, et al. (1999) Acute exercise increases nitric oxide synthase activity in skeletal muscle. Am J Physiol Endocrinol Metab 277, E390-E394.

53. Lamprecht M, Greilberger JF, Schwaberger G, et al. (2008) Single bouts of exercise affects albumin redox state and carbonyl groups on plasma protein of trained men in a workload-dependent manner. J Appl Physiol 104, 1611-1617.

54. Young DS (1987) Implementation of SI units for clinical laboratory tables - style specifications and conversion tables. Ann Intern Med 106, 114-129.

55. Deutsche Gesellschaft für Ernährung (DGE), Österreichische Gesellschaft für Ernährung (ÖGE), Schweizerische Vereinigung für Ernährung (SVE) (2008) Referenzwerte für die Nährstoffzufuhr, Frankfurt, 3. korrigierter Nachdruck der 1. Auflage, Umschau Braus GmbH (German). (German Nutrition Society, Austrian Nutrition Society, Swiss Association of Nutrition (2008) Reference Values for Nutrient Intake, 3rd revision of the 1st issue. Frankfurt: Umschau Braus Limited). 